\title{
A double-blind placebo-controlled trial of minocycline on translocator protein distribution volume in treatment-resistant major depressive disorder
}

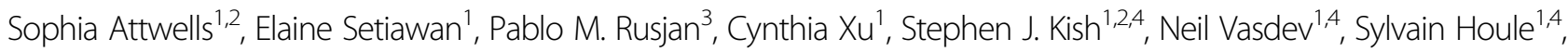
Apitharani Santhirakumar ${ }^{1}$ and Jeffrey H. Meyer (B) ${ }^{1,2,4}$

\begin{abstract}
Gliosis is implicated in the pathophysiology of many neuropsychiatric diseases, including treatment-resistant major depressive disorder (TRD). Translocator protein total distribution volume (TSPO $\mathrm{V}_{\mathrm{T}}$ ), a brain marker mainly reflective of gliosis in disease, can be measured using positron emission tomography (PET). Minocycline reduces gliosis and translocator protein binding in rodents, but this is not established in humans. Here, the ability of oral minocycline to reduce TSPO $V_{T}$ was assessed in TRD. To determine whether oral minocycline, as compared to placebo, can reduce prefrontal cortex (PFC), anterior cingulate cortex (ACC), and insula TSPO $V_{T}$ in TRD, twenty-one TRD participants underwent two $\left[{ }^{18}\right.$ F]FEPPA PET scans to measure TSPO $V_{\mathrm{T}}$. These were completed before and after either oral minocycline $100 \mathrm{mg}$ bid or placebo which was administered in a randomized double-blinded fashion for 8 weeks. There was no significant difference between the minocycline and placebo groups on change in TSPO $V_{T}$ within the PFC, ACC, and insula (repeated measures ANOVA, effect of group interaction, PFC: $F_{1,19}=0.28, P=0.60$; $A C C: F_{1,19}=$ $0.54, P=0.47$; insula $F_{1,19}=1.6, P=0.22$ ). Oral minocycline had no significant effect on TSPO $V_{T}$ which suggests that this dosage is insufficient to reduce gliosis in TRD. To target gliosis in TRD either alternative therapeutics or intravenous formulations of minocycline should be investigated. These results also suggest that across neuropsychiatric diseases in humans, it should be assumed that oral minocycline will not reduce TSPO $V_{T}$ or gliosis unless empirically demonstrated.
\end{abstract}

\section{Introduction}

In the pathophysiology of several burdensome, frequently treatment-resistant neuropsychiatric diseases, including Alzheimer's disease, Parkinson's disease, Huntington's disease, obsessive compulsive disorder, and major depressive disorder (MDD), an inflammatory response of a gliosis composed of activation and

\footnotetext{
Correspondence: Jeffrey H. Meyer (jeffrey.meyer@utoronto.ca)

${ }^{1}$ Brain Health Imaging Centre and Campbell Family Mental Health Research Institute at the Centre for Addiction and Mental Health, 250 College Street, Toronto, ON M5T 1R8, Canada

Department of Pharmacology and Toxicology, University of Toronto, 1 King's College Circle, Toronto, ON M5S 1A8, Canada
}

Full list of author information is available at the end of the article proliferation of microglia and/or astroglia is implicated ${ }^{1,2}$. Presently there is no established intervention for targeting this gliosis in humans, but minocycline reduces brain gliosis in rodent and in vitro studies. Minocycline is a second-generation tetracycline antibiotic with off target anti-inflammatory effects. The anti-inflammatory effects include lowering of lipopolysaccharide (LPS) induced brain expression of major histocompatibility complex II, tumor necrosis factor a, interferon $\gamma$, interleukin 6 , and interleukin IL-1 $\beta$ mRNA and/or protein; and reduction of microglial and astroglial activation and proliferation in animal disease models of neurodegeneration, traumatic brain injury, and sickness behavior ${ }^{3-7}$. Although these

\section{(c) The Author(s) 2021}

\footnotetext{
(c) Open Access This article is licensed under a Creative Commons Attribution 4.0 International License, which permits use, sharing, adaptation, distribution and reproduction in any medium or format, as long as you give appropriate credit to the original author(s) and the source, provide a link to the Creative Commons license, and indicate if changes were made. The images or other third party material in this article are included in the article's Creative Commons license, unless indicated otherwise in a credit line to the material. If material is not included in the article's Creative Commons license and your intended use is not permitted by statutory regulation or exceeds the permitted use, you will need to obtain permission directly from the copyright holder. To view a copy of this license, visit http://creativecommons.org/licenses/by/4.0/.
} 
models suggest that minocycline has an important translational application to target gliosis, it has not been definitively demonstrated that minocycline affects gliosis in human neuropsychiatric diseases.

Translocator protein (TSPO) is expressed in activated microglia, activated astroglia, and endothelial cells. In rodent models of gliosis after LPS administration, toxin, and stroke, overall TSPO binding is strongly related to its magnitude of expression in activated microglia ${ }^{8-10}$. In human neuropsychiatric illnesses, such as AD, HIV encephalitis, multiple sclerosis, amyotrophic lateral sclerosis, frontotemporal dementia, and stroke, TSPO is expressed mainly in activated microglia and to a lesser extent in astroglia ${ }^{11-13}$. In health, most TSPO expression is consequent to its presence in endothelial cells. Thus the differential elevation of TSPO binding in neuropsychiatric disease as compared to health is usually attributed to the greater activation and proliferation of TSPO-positive microglia and astroglia that occur during gliosis ${ }^{2,14}$.

Minocycline reduces TSPO binding in rodents with gliosis but in humans there has been minimal study. In rats, the heightened TSPO binding associated with conditions that increase microglial and astroglial activation like middle cerebral artery occlusion, toll-like receptor 2 agonist exposure, and pilocarpine/kainate-induced status epilepticus are reduced by minocycline administration with substantial effects of $30-50 \%{ }^{15-17}$. The first clinical trial of minocycline with TSPO PET imaging in humans had a sample size too small to be analyzed statistically with only three cases of multisystem atrophy receiving minocycline ${ }^{18}$. The other trial evaluating the effect of oral minocycline on TSPO binding in humans was conducted in traumatic brain injury and it compared the effect of minocycline in seven cases to placebo in five cases, finding a significant $20 \%$ reduction of TSPO distribution volume $\left(\mathrm{TSPO} \mathrm{V}_{\mathrm{T}}\right.$ ), an index of TSPO density, in gray matter and white matter regions as measured with $\left[{ }^{11} \mathrm{C}\right]$ PBR28 $\mathrm{PET}^{19}$. The magnitude of effect in the latter study is promising. However, it would have been ideal to have included a few more cases in each group and it is unclear whether this result is generalizable to other psychiatric diseases.

In the present study, we evaluated the effect of minocycline on TSPO $V_{T}$ as measured with $\left[{ }^{18} \mathrm{~F}\right]$ FEPPA PET in treatment-resistant major depressive episodes (MDE) of MDD ${ }^{14}$. $\left[{ }^{18} \mathrm{~F}\right] \mathrm{FEPPA}$ has excellent properties including high, selective affinity for $\mathrm{TSPO}^{20}$, increased binding during induced neuroinflammation ${ }^{21}$, no detectable radioactive metabolites in brain in preclinical assessment $^{22}$ and a high ratio of specific binding relative to free and non-specific binding ${ }^{22,23}$. TSPO PET imaging studies of MDE from MDD consistently report greater TSPO $V_{T}$, or similar measures of TSPO binding, in the prefrontal cortex (PFC) and anterior cingulate cortex (ACC), as demonstrated across six different studies at four different sites totaling 142 patients and 93 controls $^{24-28}$, with ACC values elevated $15-67 \%$ and PFC values elevated $25-35 \%$. One additional $\left[{ }^{11} \mathrm{C}\right]$ PBR28 study reported a negative result within a sample combining five MDE subjects and four recovered depressed subjects, however, the same group reports that the five subjects in a current $\mathrm{MDE}$ also had elevated TSPO $V_{T}$ values in gray-matter regions ${ }^{29}$.

Two double-blind, randomized placebo-controlled trials of minocycline demonstrated promising effects on MDE with one trial demonstrating a significant reduction on core symptoms and the other reporting trend reductions in anxiety ${ }^{30,31}$. Therefore, given that minocycline reduces gliosis $^{3-7}$ and TSPO binding in rodent models ${ }^{15-17}$, that there is some evidence of symptom reduction in doubleblind placebo-controlled clinical trials of minocycline in $\mathrm{MDE}^{30,31}$, and that TSPO $V_{\mathrm{T}}$ is consistently elevated in the PFC, ACC and insula during $\mathrm{MDE}^{24-28}$, it was hypothesized that minocycline would lower TSPO $V_{\mathrm{T}}$ in the PFC, ACC, and insula in MDE in humans. The PFC and ACC were prioritized regions of interest (ROIs) because neuropathological abnormalities are frequently identified within these regions in MDE, subregions within these structures regulate affect, and these regions are functionally sensitive to depressed mood induction ${ }^{32}$. In addition, the insula was prioritized because it is implicated in mediating some of the sickness behaviors in MDD: The insula participates in homeostatic regulation and interoceptive signaling; ${ }^{33,34}$ and has elevated TSPO $\mathrm{V}_{\mathrm{T}}$ during $\mathrm{MDE}^{27,28}$.

\section{Materials, participants, and methods Participants}

Twenty-three participants with treatment-resistant MDE secondary to MDD were recruited from the Greater Toronto Area and a tertiary psychiatric hospital, Centre for Addiction and Mental Health (CAMH), between February 2015 and March 2019 (Fig. 1, Table 1). Inclusion criteria included age 18-65; a positive diagnosis of MDE based on the Structured Clinical Interview for DSM-IV with confirmation by a consultation with a psychiatrist (J.H.M.) and a minimum score of 19 on the 17 item Hamilton Depression Rating Scale (HDRS) at screening ${ }^{35}$. TRD was defined as a history of nonresponse to a clinically appropriate dose and duration of at least one antidepressant whose mechanism of action includes raising extracellular serotonin concentration and one or more antidepressants whose mechanism of action includes raising norepinephrine concentration; or nonresponse to at least one serotonin and norepinephrine reuptake inhibitor. All participants confirmed receiving a stable clinical dose of an antidepressant medication for at least 4 weeks prior to PET scanning (see Supplementary Table 1). 


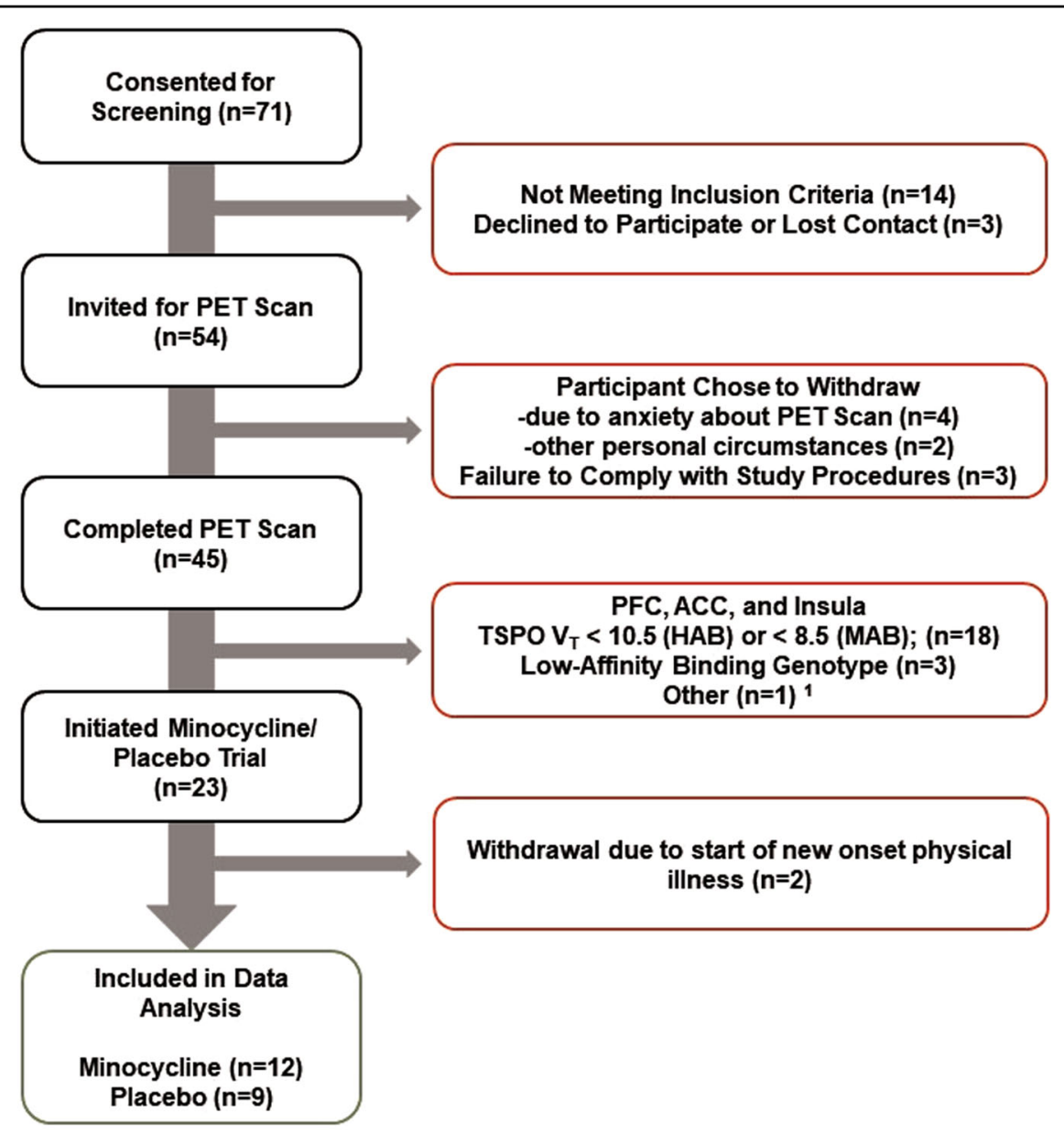

Fig. 1 Flow diagram of study participants. Flow of participants through the study. ${ }^{1}$ Diagnosis reclassified as bipolar type II. ACC anterior cingulate cortex, PFC prefrontal cortex, PET positron emission tomography, TSPO $\mathrm{V}_{\mathrm{T}}$ translocator protein total distribution volume.

Participants were excluded if they had a history of, or had concurrent alcohol or substance dependence. This was corroborated by urine drug testing at screening and on the PET scan day. In addition, to avoid potential biases from recent infection, all were free from acute medical illnesses for the 2 weeks before PET scanning. Also, participants were excluded if they had used brain stimulation treatments within the 6 months before scanning, had used anti-inflammatory drugs lasting at least 1 week within the past month, or were taking hormone replacement therapy. Other exclusion criteria were bipolar disorder (type I or II) and antisocial personality disorder. No participant had a history of neurological illness, autoimmune disorder, severe hepatic or renal disease, gastrointestinal disease, ischaemic heart disease, cerebrovascular disorder, or congestive heart failure. Participants were also excluded if they were pregnant or breastfeeding; and all women received a urine pregnancy test on the PET scan day.

Like all second-generation TSPO radioligands, the binding affinity of $\left[{ }^{18} \mathrm{~F}\right] \mathrm{FEPPA}$ for TSPO is affected by a single-nucleotide polymorphism (rs6971; $\mathrm{C} \rightarrow \mathrm{T}$ ) in exon 4 of the TSPO gene (NCBI Entrez Gene 706) ${ }^{36,37}$. Highaffinity binders (HAB) express a single high-affinity binding site for TSPO, whereas mixed-affinity binders (MAB) express in equal quantities both high-affinity and low-affinity binding sites for TSPO. Previous studies demonstrate the rs6971 polymorphism to be a significant predictor of $\left[{ }^{18} \mathrm{~F}\right]$ FEPPA TSPO $\mathrm{V}_{\mathrm{T}}$ in human brains ${ }^{36}$. Individuals with high-affinity binding (Ala147/Ala147) and mixed-affinity binding (Ala147/Thr147) account for more than $95 \%$ of the population in the Greater Toronto Area $^{28,36}$. Intravenous whole blood was collected and the rs6971 polymorphism was genotyped (see Supplementary Information). Since the translational aim for the clinical setting was to develop minocycline for those with more prominent gliosis, an additional inclusion criterion was that TSPO $\mathrm{V}_{\mathrm{T}}$ was at least above 10.5 for $\mathrm{HAB}$ and 8.5 for $\mathrm{MAB}$ in any of the primary ROIs (PFC, ACC, insula). By applying such a threshold, it was anticipated that the resulting TSPO $\mathrm{V}_{\mathrm{T}}$ values in the sample gathered would 
Table 1 Demographic characteristics of study participants.

\begin{tabular}{|c|c|c|c|}
\hline Characteristic & $\begin{array}{l}\text { Minocycline } \\
(n=12)\end{array}$ & $\begin{array}{l}\text { Placebo } \\
(n=9)\end{array}$ & $P$ value \\
\hline Sex & & & $0.66^{\mathrm{a}}$ \\
\hline Female & 8 & 7 & \\
\hline Male & 4 & 2 & \\
\hline Translocator protein genotype ${ }^{b}$ & & & $1.000^{\mathrm{a}}$ \\
\hline High-affinity binders & 9 & 6 & \\
\hline Mixed-affinity binders & 3 & 3 & \\
\hline Age, years, mean (SD) & $36.5(13.4)$ & $36.9(12.0)$ & $0.94^{c}$ \\
\hline Body mass index, mean (SD) & $24.7(2.7)$ & $25.1(4.3)$ & $0.76^{c}$ \\
\hline $\begin{array}{l}\text { Level of education, years, } \\
\text { mean (SD) }\end{array}$ & $17.3(4.0)$ & $15.3(2.3)$ & $0.22^{c}$ \\
\hline HDRS score ${ }^{d}$ & $20.2(3.5)$ & $20.3(6.1)$ & $0.94^{c}$ \\
\hline Age at first MDE, years, mean (SD) & $14.8(6.3)$ & $20.4(11.0)$ & $0.15^{c}$ \\
\hline Number of MDEs, mean (SD) & $5.4(3.4)$ & $8.0(5.6)$ & $0.21^{\mathrm{c}}$ \\
\hline $\begin{array}{l}\text { Number of previous antidepressant } \\
\text { treatment, mean }(S D)^{e}\end{array}$ & $5.0(3.4)$ & $5.6(2.0)$ & $0.67^{c}$ \\
\hline $\begin{array}{l}\text { Previous antidepressant treatment, } \\
\text { years, mean (SD) }\end{array}$ & $9.2(7.1)$ & $7.3(6.5)$ & $0.53^{c}$ \\
\hline $\begin{array}{l}\text { Years of untreated major } \\
\text { depressive disorder, mean (SD) }\end{array}$ & $11.9(10.5)$ & $8.9(8.2)$ & $0.49^{c}$ \\
\hline $\begin{array}{l}\text { Number of previous suicide } \\
\text { attempts, mean (SD) }\end{array}$ & $0.3(0.6)$ & $0.4(0.7)$ & $0.52^{c}$ \\
\hline
\end{tabular}

HDRS Hamilton Depression Rating Scale, MDE major depressive episode, PET positron emission tomography, $S D$ standard deviation, $\left[{ }^{18} \mathrm{~F}\right] \mathrm{FEPPA}, \mathrm{F}$ 18-labeled $\mathrm{N}$-(2-(2-fluoroethoxy)benzyl)-N-(4-phenoxypyridin-3-yl)acetamide).

${ }^{\mathrm{a}}$ Fisher's exact test.

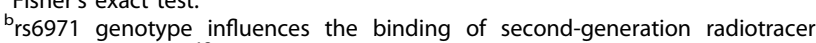
ligands including $\left[{ }^{18} \mathrm{~F}\right] \mathrm{FEPPA}$.

'Univariate analysis of variance.

${ }^{d}$ Score derived at baseline PET scan. At the time of initial screening, HDRS score was 19 or greater but with ongoing variation in severity of illness, five participants had HDRS below 19 at the time of the first PET scan.

ePrevious treatments included quetiapine, repetitive transcranial magnetic stimulation, magnetic seizure therapy, and electroconvulsive therapy.

ensure recruitment of MDE participants with TSPO $\mathrm{V}_{\mathrm{T}}$ values higher than most healthy people ${ }^{28}$. Homozygotes for the low-affinity binding gene (Thr147/Thr147) were withdrawn from the study.

This study was approved by the Research Ethics Board of the CAMH and was therefore performed in accordance with the ethical standards established in the 1964 Declaration of Helsinki. All participants gave their informed consent prior to their inclusion in the study. All details that might disclose the identity of the participants have been omitted.

\section{Image acquisition and analysis}

Each participant underwent two $\left[{ }^{18} \mathrm{~F}\right]$ FEPPA PET scans (HRRT; CPS/Siemens, Knoxville, TN, USA): one scan pre minocycline-placebo treatment and one scan post 8-week minocycline-placebo treatment. Intravenous $\left[{ }^{18} \mathrm{~F}\right]$ FEPPA was administered as a bolus (PET Scan 1: mean [standard deviation (SD)], 188.2 [12.5] MBq; PET scan 2: mean [SD], $186.0[12.5] \mathrm{MBq})$. The $\left[{ }^{18} \mathrm{~F}\right] \mathrm{FEPPA}$ was of high radiochemical purity (PET scan 1: >99.0\%; PET scan 2: 99.3\%) and high specific activity (PET scan 1: mean [SD], 65.8 [41.3] TBq/mmol; PET scan 2: mean [SD], 145.0 [146.2] $\mathrm{TBq} / \mathrm{mmol}$ (no relationship between TSPO $\mathrm{V}_{\mathrm{T}}$ and specific activity; $R^{2}<0.012$; see Supplementary Results). The PET scan duration was 125 min after the injection of $\left[{ }^{18} \mathrm{~F}\right]$ FEPPA (see Supplementary Information).

Primary ROIs were the PFC, ACC, and insula. Additional regions sampled included subregions of the PFC (medial, ventrolateral, dorsolateral, and orbitofrontal), temporal cortex, parietal cortex, occipital cortex, thalamus, dorsal putamen, dorsal caudate, ventral striatum, and hippocampus. ROIs were defined as previously described $^{38}$. ROIs were delineated on proton density magnetic resonance imaging scans (Signa 3-T MRI scanner, General Electric, Milwaukee, WI, USA; section thickness $2 \mathrm{~mm}$, repetition time $6000 \mathrm{~ms}$, echo time $8 \mathrm{~ms}$, flip angle $90^{\circ}$, one excitation, acquisition matrix $256 \times 192$, and field of view $16.5 \mathrm{~cm}$ ) using the in-house software, Regions of Mental Interest. Arterial sampling was conducted to determine the input to a two tissue compartment model to measure TSPO $V_{\mathrm{T}}$ (see Supplementary Information).

\section{Minocycline dosing regime and compliance calculation}

All participants received either minocycline or placebo for 8 weeks in a randomized, double-blind, placebocontrolled trial. Randomization was performed using an algorithm created in R Version 3.1.0 in February 2015. The algorithm consisted of a 1:1 ratio of active vs. placebo. Bloc randomization was used (bloc size $=20$ ), the total number of codes was 80 , and drop outs were not replaced. For placebo, the number and appearance of the pills were identical to minocycline. Dosing of minocycline was $50 \mathrm{mg}$ per day on week $1,50 \mathrm{mg}$ bid on week 2 , and $100 \mathrm{mg}$ bid on weeks $3-8$. Minocycline taken $100 \mathrm{mg}$ bid is well tolerated in human clinical trials ${ }^{30,31}$ and was the same dose given by Scott et al. which lowered TSPO $V_{\mathrm{T}}$ in traumatic brain injury ${ }^{19}$. For tapering, dosing was reduced to $50 \mathrm{mg}$ bid for a week, and then stopped. The blind was broken following the completion of 20 participants and 1 additional participant completed the study in a blinded state. Participants attended follow-up appointments with the treating psychiatrist and research staff at weeks 2 , $4-5$, and 8 .

To increase compliance, minocycline and placebo were given to the participants in blister packs. The blister packs contained designated sealed compartments for the medication to be taken at times of the day (Monday through 
Table 2 Effect of oral minocycline treatment on regional translocator protein total distribution volume.

\begin{tabular}{|c|c|c|c|c|c|c|c|c|}
\hline & \multicolumn{2}{|c|}{ Baseline TSPO $\mathrm{V}_{\mathrm{T}}$} & \multicolumn{2}{|c|}{ Post-treatment TSPO $\mathrm{V}_{\mathrm{T}}$} & \multicolumn{4}{|c|}{${ }^{\mathrm{a}}$ Change in TSPO $\mathrm{V}_{\mathrm{T}}$} \\
\hline & \multirow{2}{*}{ 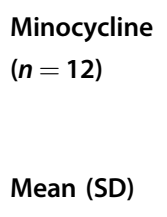 } & \multirow{2}{*}{ 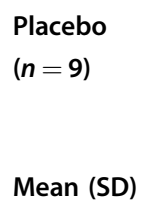 } & \multirow{2}{*}{ 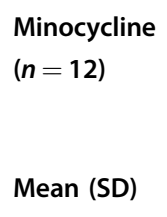 } & \multirow{2}{*}{ 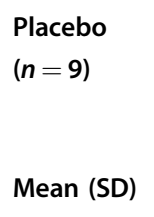 } & \multirow{2}{*}{$\begin{array}{l}\text { Minocycline } \\
(n=12) \\
\text { Mean (SD) }\end{array}$} & \multirow{2}{*}{ 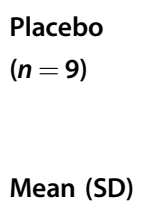 } & \multicolumn{2}{|c|}{$\begin{array}{l}\text { Effect of } \\
\text { treatment } \\
\text { group }^{b}\end{array}$} \\
\hline & & & & & & & $F_{1,19}$ & $P$ value \\
\hline PFC & $12.4(2.6)$ & $13.6(4.9)$ & $11.8(3.6)$ & $12.5(3.7)$ & $-0.61(1.7)$ & $-1.1(2.5)$ & 0.28 & 0.60 \\
\hline MPFC & $12.2(2.6)$ & $13.3(5.1)$ & $11.3(3.8)$ & $11.9(3.7)$ & $-0.92(1.9)$ & $-1.4(2.3)$ & 0.24 & 0.63 \\
\hline VLPFC & $13.2(2.5)$ & $14.6(5.0)$ & $12.8(4.0)$ & $13.5(4.2)$ & $-0.38(2.0)$ & $-1.2(2.7)$ & 0.58 & 0.46 \\
\hline DLPFC & $12.4(2.7)$ & $13.4(4.7)$ & $11.8(3.5)$ & $12.4(3.7)$ & $-0.58(1.8)$ & $-0.97(2.3)$ & 0.19 & 0.67 \\
\hline OFC & $12.3(3.4)$ & $14.1(4.3)$ & $11.4(3.9)$ & $12.0(3.4)$ & $-0.83(2.0)$ & $-2.1(2.4)$ & 1.8 & 0.19 \\
\hline ACC & $12.1(3.0)$ & $13.2(5.0)$ & $11.5(4.4)$ & $11.9(3.3)$ & $-0.51(2.8)$ & $-1.3(2.6)$ & 0.54 & 0.47 \\
\hline Insula & $12.9(3.0)$ & $14.1(4.7)$ & $12.0(3.9)$ & $12.1(3.8)$ & $-0.93(1.7)$ & $-2.0(2.2)$ & 1.6 & 0.22 \\
\hline Temporal cortex & $12.6(3.0)$ & $13.8(5.0)$ & $12.1(4.1)$ & $12.1(3.4)$ & $-0.53(1.6)$ & $-1.8(2.2)$ & 2.3 & 0.15 \\
\hline Parietal cortex & $13.2(3.0)$ & $14.6(5.4)$ & $13.0(3.9)$ & $13.2(4.2)$ & $-0.21(1.9)$ & $-1.4(2.3)$ & 1.7 & 0.21 \\
\hline Occipital cortex & $12.7(3.0)$ & $13.8(5.1)$ & $12.0(4.5)$ & $12.2(3.8)$ & $-0.69(1.8)$ & $-1.6(2.6)$ & 0.94 & 0.35 \\
\hline Thalamus & $15.1(3.8)$ & $16.0(5.3)$ & $14.2(5.3)$ & $13.8(4.4)$ & $-0.89(2.5)$ & $-2.2(3.4)$ & 0.99 & 0.33 \\
\hline Dorsal putamen & $10.9(2.8)$ & $11.7(4.1)$ & $10.2(3.4)$ & $10.2(3.1)$ & $-0.77(1.7)$ & $-1.5(2.1)$ & 0.72 & 0.41 \\
\hline Dorsal caudate & $10.0(2.4)$ & $11.1(3.9)$ & $9.8(3.2)$ & $9.8(3.4)$ & $-0.25(2.2)$ & $-1.3(2.7)$ & 0.95 & 0.34 \\
\hline Ventral striatum & $11.8(3.3)$ & $12.1(4.8)$ & $10.7(3.9)$ & $10.9(3.2)$ & $-1.1(2.3)$ & $-1.2(2.6)$ & 0.02 & 0.89 \\
\hline Hippocampus & $11.2(3.0)$ & $13.1(5.3)$ & $11.4(4.6)$ & $11.6(3.3)$ & $0.20(3.4)$ & $-1.5(5.1)$ & 0.81 & 0.38 \\
\hline
\end{tabular}

ACC anterior cingulate cortex, DLPFC dorsolateral prefrontal cortex, MPFC medial prefrontal cortex, OFC orbitofrontal cortex, PFC prefrontal cortex, PET positron emission tomography, $S D$ standard deviation, TSPO $V_{T}$ translocator protein total distribution volume, VLPFC ventrolateral prefrontal cortex.

${ }^{a}$ Change in TSPO $V_{T}$ was calculated as regional TSPO $V_{T}$ post-treatment minus regional TSPO $V_{T}$ baseline. Analysis of variance comparing baseline TSPO $V_{T}$ values found no significant difference between groups for any regions (effects of group and genotype included, effect of group, $F_{1,18}=3.4-0.27, P=0.081-0.61$ ).

${ }^{\mathrm{b}}$ Repeated measures analysis of variance evaluating effect of group interaction on repeated measure of TSPO $\mathrm{V}_{\mathrm{T}}$.

Sunday, breakfast and supper). To calculate compliance, participants informed study staff if they missed a dosage. In addition, blister packs were collected after use, and remaining pills were counted and returned to the CAMH pharmacy. Compliance was recorded in a subset of 14 subjects and average compliance was calculated to be 99.3\% $(\mathrm{SD}=1.6)$.

\section{Mood questionnaires}

The 17 item HDRS was completed by participants during all study visits ${ }^{35}$. Baseline scores were taken from the day at which minocycline or placebo was initiated. Participants returned to the CAMH for follow-up visits at weeks 2 and $4-5$. The second $\left[{ }^{18} \mathrm{~F}\right]$ FEPPA PET scan took place during week 8 of minocycline or placebo treatment.

\section{Statistical analysis}

For the primary analyses, a repeated measures analysis of variance (rmANOVA) was applied. One repeated measure was TSPO $V_{T}$ within each region before and after the intervention (minocycline or placebo), a measure to assess change over the time of the intervention. The other repeated measure was region (PFC, ACC, or insula) which was sampled concurrently at each timepoint within each participant. Hence the within group factors were region and scan time (first or second PET scan) and the between group factors were minocycline or placebo with the interaction between scan time and group being the key analysis. Similar secondary analyses were conducted with a rmANOVA for each region, with regional TSPO $\mathrm{V}_{\mathrm{T}}$ as the dependent variable, scan time and group as the predictor variables, assessing the interaction between scan time and group. As an additional analysis, a mixed effects model was applied in which participants are considered random effects; brain regions and scan time are withinsubject fixed effects; group (minocycline or placebo) is a between subject fixed effect; and the interaction between visit and group was assessed.

Additional exploratory analyses were conducted to assess the relationship between change in the severity of $\mathrm{MDE}$ and change in TSPO $\mathrm{V}_{\mathrm{T}}$ within the PFC, ACC, and insula. The two TSPO $V_{T}$ values for each region from the 


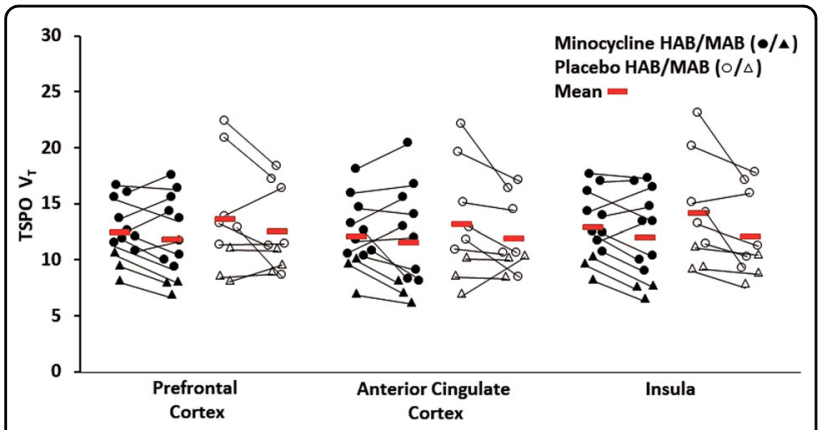

Fig. 2 Effect of oral minocycline treatment on translocator protein total distribution volume in participants with treatmentresistant major depressive disorder. There were no significant differences in prefrontal cortex, anterior cingulate cortex, or insula TSPO $V_{T}$ between pre- and post-minocycline treatment in the group receiving minocycline as compared to the group receiving placebo (repeated measures ANOVA, effect of group interaction, PFC: $F_{1,19}=$ $0.28, P=0.60$; ACC: $F_{1,19}=0.54, P=0.47$; insula $F_{1,19}=1.6, P=0.22$ ). All second-generation TSPO radioligands, such as $\left[{ }^{18} \mathrm{~F}\right] \mathrm{FEPPA}$, show differential binding according to the single-nucleotide polymorphism rs6971 of the TSPO gene, resulting in high-affinity binders (circles) and mixed-affinity binders (triangles). TSPO $V_{T}$ values represent raw values unadjusted for genotype. ANOVA analysis of variance, HAB highaffinity binder, MAB mixed-affinity binder, $\mathrm{TSPO}_{\mathrm{T}}$ translocator protein total distribution volume, $\left[{ }^{18} \mathrm{~F}\right] \mathrm{FEPPA}, \mathrm{F}$ 18-labeled $\mathrm{N}-(2-(2-$ fluoroethoxy)benzyl)-N-(4-phenoxypyridin-3-yl)acetamide).

first and second $\left[{ }^{18} \mathrm{~F}\right]$ FEPPA PET scans were the repeated dependent variables and then the change in HDRS and the rs6971 genotype were applied as the independent variables, prioritizing the interactions between change in HDRS and scan time.

\section{Results}

\section{Participant demographics and measures at baseline}

Overall, 23 TRD eligible participants were recruited and 21 completed the 8-week trial (Fig. 1). Twelve participants received minocycline and nine received placebo (Table 1). The mean and SD of the age of the participants, age of first MDE onset, and number of previous non-responses to treatment were $36.7 \pm 12.5,17.2 \pm 8.8$, and $5.2 \pm 2.8$, respectively. Assigned sex at birth of participants included 15 females and 6 males. Preferred genders of participants included 15 females and 6 males. Age, sex, rs6971 genotype, BMI, and HDRS score at the time of the first PET scan were similar across groups as were other demographic characteristics (Table 1). Baseline TSPO $\mathrm{V}_{\mathrm{T}}$ values in all regions assessed were similar between the minocycline and placebo groups (analysis of variance (ANOVA) $, \quad F_{1,18}=3.4-0.27, \quad P=0.081-0.61 ; \quad$ baseline TSPO $V_{T}$ values displayed in Table 2).

No effect of minocycline vs. placebo on change in TSPO $V_{T}$

There was no differential effect between the minocycline and placebo groups on change in TSPO $V_{\mathrm{T}}$ across the first and second PET scans (scan time) in the PFC, ACC, and insula (rmANOVA; first or second TSPO $V_{T}$ and region were repeated dependent variables with first or second TSPO $\mathrm{V}_{\mathrm{T}}$ reflecting the effect of scan time; interaction effect of group on scan time; $F_{1,19}=0.79, P=$ 0.39). Nor was there a differential effect between minocycline and placebo groups on change in TSPO $V_{T}$ over time within individual regions. (rmANOVA; first or second TSPO $V_{T}$ repeated dependent variables analyzed within each region, PFC: $F_{1,19}=0.28, P=0.60$; ACC: $F_{1,19}$ $=0.54, P=0.47$; insula $F_{1,19}=1.6, P=0.22$ ); Fig. 2 ). When genotype was added as an additional independent variable it was not significant as a predictor of the repeated measure and then not included in the analyses. There was also no differential effect of minocycline vs. placebo on change in TSPO $\mathrm{V}_{\mathrm{T}}$ in any other region (rmANOVA, applied for each region, first or second TSPO $V_{T}$ was the repeated dependent variable reflecting scan time, interaction of group on change in TSPO $\mathrm{V}_{\mathrm{T}} ; F_{1,19}=2.3-0.02$, $P=0.15-0.89$; Table 2). When genotype was added as an additional independent variable it was not significant as a predictor of the repeated measure and then not included in the analyses. In addition, when a mixed effects model was applied, with participant as the random effect, repeated scan as a fixed effect, region as a fixed effect, and group as a fixed effect, there was no significant interactive effect between the effects of repeated scan and group $(P=0.39)$.

\section{No significant relationship between change in $\mathrm{TSPO}_{\mathrm{T}}$ and change in hamilton depression rating scale scores}

Mean change in HDRS was modest in both groups (mean [SD]: minocycline -5.7 [7.6]; placebo -7.1 [5.6]; with three participants in the placebo group and two participants in the minocycline group responding (HDRS decrease $\geq 50 \%$ ), and two participants in the placebo group and one participant in the minocycline group remitting (final HDRS $\leq 7$ ). There was no significant difference in the reduction in HDRS between groups (mixed effects model, $P=0.64$, see Supplementary Information)

There was no relationship between change in the severity of MDE and change in TSPO $\mathrm{V}_{\mathrm{T}}$ within the PFC, $\mathrm{ACC}$, and insula (rmANOVA for each region; scan time of first or second TSPO $\mathrm{V}_{\mathrm{T}}$ were repeated dependent variables; effect of HDRS $F_{1,19}=0.66-0.06, P=0.43-0.82$; Fig. 3). Additional analyses assessing the interaction of HDRS and genotype or inclusion of genotype were similarly nonsignificant. Repeating the same analysis within the minocycline group alone similarly found no relationship between severity of MDE and change in TSPO $V_{T}$ (rmANOVA for each region; scan time of first or second TSPO $\mathrm{V}_{\mathrm{T}}$ were repeated dependent variables; effect of HDRS $\left.\quad F_{1,10}=0.54-0.02, \quad P=0.48-0.89\right)$. Additional 

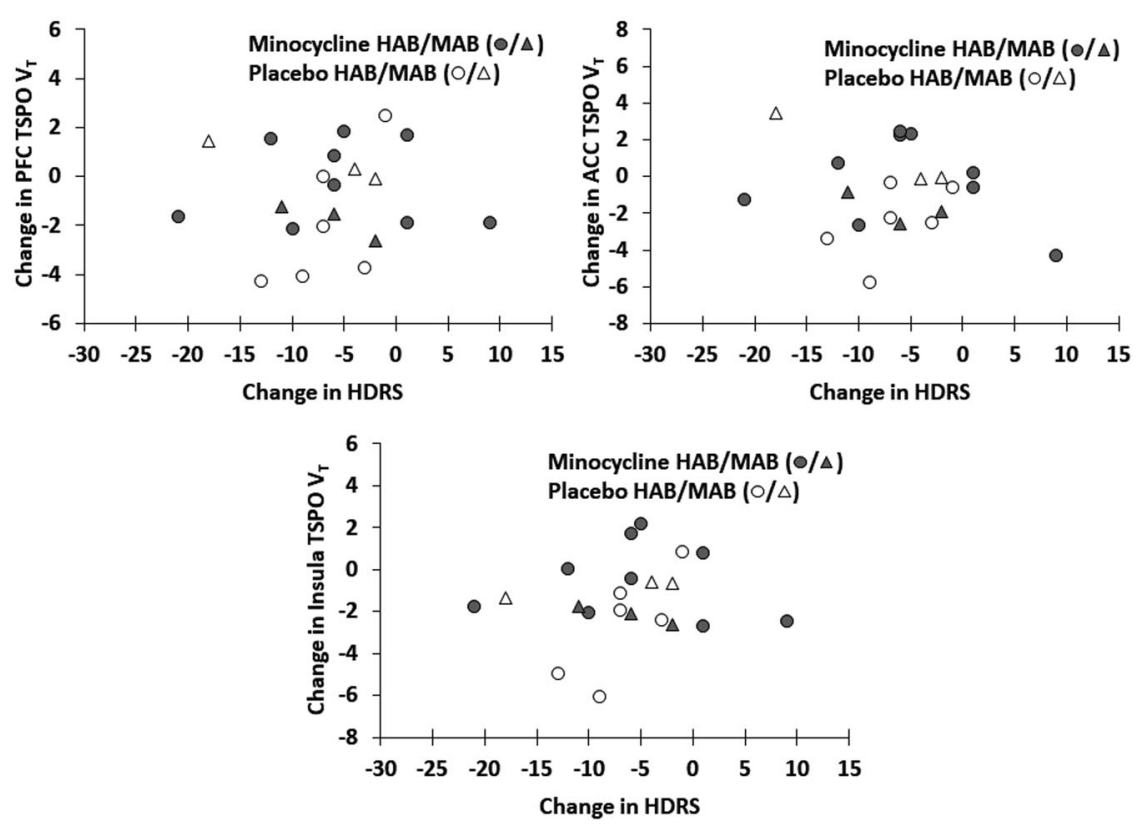

Fig. 3 Relationship between change in regional translocator protein total distribution volume and change in hamilton depression rating scale scores. There were no significant relationships between the change in prefrontal cortex, anterior cingulate cortex, or insula TSPO $V_{T}$ with change in HDRS scores (repeated measures ANOVA, TSPO $V_{T}$ as dependent variable, effect of HDRS change as covariate, $F_{1,19}=0.66-0.06, P=$ 0.43-0.82). Similarly, nonsignificant effects were observed within the minocycline treated group alone. ACC anterior cingulate cortex, HAB highaffinity binder, HDRS Hamilton Depression Rating Scale, MAB mixed-affinity binder, PFC prefrontal cortex, TSPO $V_{T}$ translocator protein total distribution volume.

analyses assessing the interaction of HDRS and genotype or inclusion of genotype were similarly nonsignificant.

\section{Discussion}

This study demonstrated no significant effect of minocycline as compared to placebo on TSPO $\mathrm{V}_{\mathrm{T}}$ in the PFC, ACC, insula, or any other brain region assayed in treatment-resistant MDE. This has important implications for repurposing minocycline both in MDD and in human neuropsychiatric diseases. While the results appear to differ from previous rodent studies and the one previous study in humans, this may be accounted for by differences in the experimental conditions and/or participants, demonstrating the importance of empirical testing of target engagement.

The lack of effect of minocycline on TSPO $\mathrm{V}_{\mathrm{T}}$ may be interpreted as a lack of effect on TSPO density, which, in neuropsychiatric diseases mainly reflects gliosis with some additional contribution from endothelial cells ${ }^{2,14}$. This argues that minocycline had no effect on these sources of TSPO density in the brain at a dose of $100 \mathrm{mg}$ bid, which is considered a maximum well-tolerated oral $\operatorname{dose}^{30,31}$. This argues that repurposing minocycline for MDE in a clinical setting by administering a course of treatment with the aim of obtaining a sustained reduction in gliosis is unlikely. It also suggests the rationale for its use in clinical trials should be oriented towards alternative mechanisms like protecting against apoptosis through inhibiting caspase 1 and 3 , and upregulating $\mathrm{Bcl} 2$ expression; engagement of potential downstream effects of gliosis like inhibiting inducible nitric oxide synthase or COX-2; or other neuroprotective effects, such as scavenging peroxynitrites or inhibiting matrix metalloproteinases ${ }^{4}$.

The differences in the effect of minocycline in the present study with others may be accounted for by differences in the experimental conditions. Minocycline suppresses elevations in TSPO binding associated with gliosis and reduces gliosis itself in rodents ${ }^{15-17,39}$. However, the dosing of minocycline in such studies, is $3-30$ times larger relative to body mass ${ }^{39}$. The results of the present study also contrast the previous human data of a $20 \%$ reduction in TSPO $\mathrm{V}_{\mathrm{T}}$ reported across brain regions assessed in seven cases treated with minocycline vs. five receiving placebo, in TBI cases within 6 months of the injury $^{19}$. While the present study is almost twice as large, another factor to consider is the duration of disease with the possible entrenchment of pro-inflammatory processes contributing and maintaining the TSPO $\mathrm{V}_{\mathrm{T}}$ elevation. The mean duration of MDD illness in the present study was 20 years in contrast to a 6-month duration since TBI in the past study ${ }^{19}$ so the relative duration of illness differs by a factor as much as 40 . Given the negative results of the present study and given that conditions underlying gliosis 
in each neuropsychiatric illness differ, rather than assuming target engagement of minocycline for gliosis or TSPO $V_{\mathrm{T}}$, it should be tested within specific illnesses.

The present study sampled 21 participants and it could be speculated as to whether increasing the sample size would alter the conclusions. This is unlikely given the differential change between groups and the SD of such changes for the PFC, ACC, and insula. The resulting confidence interval indicates there is a $95 \%$ chance that the magnitude of differential reduction in TSPO $\mathrm{V}_{\mathrm{T}}$ favoring minocycline in these regions is less than $9 \%, 11 \%$, and $3 \%$, respectively. This is much smaller than the mean disease effect of MDE in these regions which is typically between 20 and 35\% and similar to other neuropsychiatric diseases ${ }^{2,40,41}$.

This study has several limitations. First, while the elevations of TSPO in neuropsychiatric illnesses are associated with greater expression in microglia with some astroglial contributions, there is some expression of TSPO in endothelial cells. However, it seems unlikely that the endothelial cell component would obscure an effect of minocycline given that in neuropsychiatric diseases and inflammatory states, TSPO expression is most prominent in glial cells ${ }^{8-13}$; the spatial extent of endothelial cells is limited since they surround blood vessels and vascular contribution to PET is considered small representing $\sim 5 \%$ of the input signal; the two tissue plus one compartment model proposed for modeling endothelial contributions is not advantageous for $\left[{ }^{18}\right.$ F]FEPPA PET ${ }^{42}$ and it seems unlikely that minocycline would evoke a strong opposite effect on TSPO expression in endothelial cells. Second, the dose of minocycline was limited to $100 \mathrm{mg}$ which is the maximum well-tolerated oral dose. Future studies could consider intravenous administration which may increase plasma levels at least twofold or alternative therapeutics, such as P2X7 inhibitors, PGE4 inhibitors, or peroxisome proliferator gamma agonists ${ }^{43-45}$. Third, TSPO participates in multiple functions including cholesterol transport, mitochondrial respiration, and apoptosis and it is possible that in future study it will be determined that there are other mechanisms which influence TSPO expression. Fourth, oral administration of minocycline might also affect the balance of the gut microbiome which has influences on bodily inflammation and its influences on brain inflammation ${ }^{46}$, which may have obscured a direct effect of minocycline. Regardless, the overall effect of oral minocycline, even if such indirect influences occurred, had a negligible effect on TSPO $V_{T}$ in brain.

In summary, to our knowledge, this is the first investigation evaluating the effect of minocycline on a measure of gliosis in the brain of humans with MDE. This is also the second study to assess the effect of minocycline on a measure of gliosis in brain in humans, applying a sample almost twice as large as the previous study which also measured TSPO $V_{T}$. There were no differences between minocycline and placebo on TSPO $\mathrm{V}_{\mathrm{T}}$. Moreover, the data argue that a meaningful reduction in TSPO $V_{T}$ with minocycline treatment, as compared to the disease effect on TSPO $V_{T}$ in MDE, is highly unlikely. The negative result of the present study also demonstrates that it cannot be assumed that the effects of minocycline on TSPO $\mathrm{V}_{\mathrm{T}}$ observed in animals in which the dosing is much higher, can be extrapolated to all human conditions. Rather, it is important to empirically test target engagement in specific neuropsychiatric illnesses as underlying disease etiologies and durations vary. Future study may consider more potent interventions, such as intravenous dosing of minocycline, or alternative therapeutics to reduce TSPO $V_{T}$ in MDE.

\section{Acknowledgements}

All experiments comply with the current laws of Canada. Alvina Ng, B.Sc.; Laura Nguyen, B.Sc.; and Colin Cole, B.Sc. worked as study PET technicians. Alan Kahn, MD and Antonio Strafella, MD provided medical coverage for the PET scans. Jun Parkes, B.Sc;; Armando Garcia, B.Sc.; and Michael Harkness, M.Sc. of Research Imaging Centre, served as PET chemistry staff. Anusha Ravichandran B.Sc.; Garfield E Detzler, B.Sc.; and Hillary Bruce, B.Sc. worked as study MRI technicians. All contributors are paid employees of the Centre for Addiction and Mental Health. All authors are paid employees of the Centre for Addiction and Mental Health. This study received funding support from the Canadian Institutes of Health Research [Canada Research Chair and Operating Grant (MOP-136955), J.H.M.; Doctoral Award (GSD-157948), S.A.; Fellowship Award, E. S.; Canada Research Chair, NV], the Brain and Behavior Foundation, and the neuroscience catalyst fund (from the Government of Ontario and Janssen). None of the funding sources participated in the execution of the project, generation of results, interpretation of data nor drafting of the manuscript. Funding for infrastructure was from the Azrieli Foundation, the Canadian Foundation for Innovation, and the Ontario Ministry for Innovation. S.H. and J. H.M. have received operating grant funds from Janssen in the past 5 years.

\section{Author details}

${ }^{1}$ Brain Health Imaging Centre and Campbell Family Mental Health Research Institute at the Centre for Addiction and Mental Health, 250 College Street, Toronto, ON M5T 1R8, Canada. ${ }^{2}$ Department of Pharmacology and Toxicology, University of Toronto, 1 King's College Circle, Toronto, ON M5S 1A8, Canada. ${ }^{3}$ Douglas Research Centre, McGill University, 6875 Boulevard Lasalle, Montreal, QC H4H 1R3, Canada. ${ }^{4}$ Department of Psychiatry, University of Toronto, 250 College Street, Toronto, ON M5T 1R8, Canada

\section{Conflict of interest}

The corresponding author (J.H.M.) has been a consultant to Lundbeck and Takeda, in the past 5 years. J.H.M. is an inventor on five patents of blood and/or clinical markers to predict brain inflammation or to diagnose affective

disorders, and a dietary supplement to reduce depressed mood postpartum. J. H.M. is receiving operating grant funding from Sanofi for a neuroinflammation PET imaging study in multiple sclerosis. J.H.M. is arranging collaborations with nutraceutical companies for the dietary supplement to prevent postpartum depression. S.K. has operating grant funds from US National Institutes of Health National Institute on Drug Abuse (DA04066) to measure microglial status in the brains of methamphetamine users, and from Jazz Pharmaceuticals for an unrelated study. All other authors declare no conflicts of interest.

\section{Publisher's note}

Springer Nature remains neutral with regard to jurisdictional claims in published maps and institutional affiliations.

Supplementary information The online version contains supplementary material available at https://doi.org/10.1038/s41398-021-01450-3.

Received: 6 July 2020 Revised: 30 March 2021 Accepted: 30 April 2021 Published online: 29 May 2021 


\section{References}

1. Hickman, S., Izzy, S., Sen, P., Morsett, L. \& El Khoury, J. Microglia in neurodegeneration. Nat. Neurosci. 21, 1359-1369 (2018).

2. Meyer, J. H. et al. Neuroinflammation in psychiatric disorders: PET imaging and promising new targets. Lancet Psychiatry 7, 1064-1074 (2020).

3. Cheng, S. et al. Minocycline reduces neuroinflammation but does not ameliorate neuron loss in a mouse model of neurodegeneration. Sci. Rep. $\mathbf{5}$, 10535 (2015)

4. Garrido-Mesa, N., Zarzuelo, A. \& Galvez, J. Minocycline: far beyond an antibiotic Br. J. Pharmacol. 169, 337-352 (2013).

5. Homsi, S. et al. Blockade of acute microglial activation by minocycline promotes neuroprotection and reduces locomotor hyperactivity after closed head injury in mice: a twelve-week follow-up study. J. Neurotrauma $\mathbf{2 7}$ 911-921 (2010).

6. Kobayashi, K. et al. Minocycline selectively inhibits M1 polarization of microglia. Cell Death Dis. 4, e525 (2013).

7. Seabrook, T. J., Jiang, L., Maier, M. \& Lemere, C. A. Minocycline affects microglia activation, abeta deposition, and behavior in APP-tg mice. Glia $\mathbf{5 3}$ 776-782 (2006)

8. Banati, R., Myers, R. \& Kreutzberg, G. W. PK ('peripheral benzodiazepine')-binding sites in the CNS indicate early and discrete brain lesions: microautoradiographic detection of [3H]PK11195 binding to activated microglia. J. Neurocytol. 26, 77-82 (1997).

9. Hannestad, J. et al. Endotoxin-induced systemic inflammation activates microglia: [(11)C]PBR28 positron emission tomography in nonhuman primates. Neurolmage 63, 232-239 (2012).

10. Martin, A. et al. Evaluation of the PBR/TSPO radioligand [(18)F]DPA-714 in a rat model of focal cerebral ischemia. J. Cereb. Blood Flow. Metab. 30, 230-241 (2010).

11. Cosenza-Nashat, M. et al. Expression of the translocator protein of $18 \mathrm{kDa}$ by microglia, macrophages and astrocytes based on immunohistochemical localization in abnormal human brain. Neuropathol. Appl. Neurobiol. 35 306-328 (2009).

12. Venneti, S., Wang, G., Nguyen, J. \& Wiley, C. A. The positron emission tomography ligand DAA1106 binds with high affinity to activated microglia in human neurological disorders. J. Neuropathol. Exp. Neurol. 67, 1001-1010 (2008).

13. Venneti, S., Wiley, C. A. \& Kofler, J. Imaging microglial activation during neuroinflammation and Alzheimer's disease. J. Neuroimmune Pharmacol. 4, 227-243 (2009)

14. Beltazar, C., Harrison-Brown, M., Middleton, R. J., Banati, R. \& Liu, G. J. Cellular sources and regional variations in the expression of the neuroinflammatory marker translocator protein (TSPO) in the normal brain. Int. J. Mol. Sci. 19, pii: E2707 (2018).

15. Converse, A. K. et al. 11C-(R)-PK11195 PET imaging of microglial activation and response to minocycline in zymosan-treated rats. J. Nucl. Med. 52, 257-262 (2011)

16. Martin, A., Boisgard, R., Kassiou, M., Dolle, F. \& Tavitian, B. Reduced PBR/TSPO expression after minocycline treatment in a rat model of focal cerebral ischemia: a PET study using [(18)F]DPA-714. Mol. Imaging Biol. 13, 10-15 (2011).

17. Wolf, B. J. et al. TSPO PET identifies different anti-inflammatory minocycline treatment response in two rodent models of epileptogenesis. Neurotherapeutics 17, 1228-1238 (2020).

18. Dodel, R. et al. Minocycline 1-year therapy in multiple-system-atrophy: effect on clinical symptoms and [(11)C] (R)-PK11195 PET (MEMSA-trial). Mov. Disord. 25, 97-107 (2010)

19. Scott, G. et al. Minocycline reduces chronic microglial activation after brain trauma but increases neurodegeneration. Brain 141, 459-471 (2018).

20. Bennacef, I. et al. Comparison of [11C]PBR28 and [18F]FEPPA as CNS peripheral benzodiazepine receptor PET ligands in the pig. J. Nucl. Med. 49, 81P (2008).

21. Kudo, G. et al. In-vivo imaging of microglial activation using a novel peripheral benzodiazepine receptor ligand, 18F-FEPPA and animal PET following 6OHDA injury of the rat striatum; a comparison with 11C-PK11195. Neurolmage 41, T94 (2008)

22. Wilson, A. A. et al. Radiosynthesis and initial evaluation of [18F]-FEPPA for PET imaging of peripheral benzodiazepine receptors. Nucl. Med. Biol. 35, 305-314 (2008).
23. Rusjan, P. M. et al. Quantitation of translocator protein binding in human brain with the novel radioligand [18F]-FEPPA and positron emission tomography. J. Cereb. Blood Flow. Metab. 31, 1807-1816 (2011).

24. Holmes, S. E. et al. Elevated translocator protein in anterior cingulate in major depression and a role for inflammation in suicidal thinking: a positron emission tomography study. Biol. Psychiatry 83, 61-69 (2018).

25. Li, H., Sagar, A. P. \& Keri, S. Translocator protein (18kDa TSPO) binding, a marker of microglia, is reduced in major depression during cognitivebehavioral therapy. Prog. Neuropsychopharmacol. Biol. Psychiatry 83, 1-7 (2018).

26. Richards, E. M. et al. PET radioligand binding to translocator protein (TSPO) is increased in unmedicated depressed subjects. EJNMMI Res. 8 57 (2018).

27. Setiawan, E. et al. Association of translocator protein total distribution volume with duration of untreated major depressive disorder: a cross-sectional study. Lancet Psychiatry 5, 339-347 (2018).

28. Setiawan, E. et al. Role of translocator protein density, a marker of neuroinflammation, in the brain during major depressive episodes. JAMA Psychiatry 72, 268-275 (2015)

29. Cosgrove, K. P. Personal communication from Hannestad et al. The neuroinflamation marker translocator protein is not elevated in individuals with mild to moderate depression: a [11C]PBR28 PET study. Brain Behav. Immun. 33 , 131-138 (2013)

30. Dean, O. M. et al. Adjunctive minocycline treatment for major depressive disorder: a proof of concept trial. Aust. N. Z. J. Psychiatry 51, 829-840 (2017).

31. Husain, M. I. et al. Minocycline as an adjunct for treatment-resistant depressive symptoms: a pilot randomised placebo-controlled trial. J. Psychopharmacol. 31, 1166-1175 (2017)

32. Ressler, K. J. \& Mayberg, H. S. Targeting abnormal neural circuits in mood and anxiety disorders: from the laboratory to the clinic. Nat. Neurosci. 10 1116-1124 (2007)

33. Avery, J. A. et al. Major depressive disorder is associated with abnormal interoceptive activity and functional connectivity in the insula. Biol. Psychiatry 76, 258-266 (2014)

34. Simmons, W. K. et al. Category-specific integration of homeostatic signals in caudal but not rostral human insula. Nat. Neurosci. 16, 1551-1552 (2013).

35. Hamilton, M. A rating scale for depression. J. Neurol. Neurosurg. Psychiatry $\mathbf{2 3}$ 56-62 (1960).

36. Mizrahi, R. et al. Translocator protein (18 kDa) polymorphism (rs6971) explains in-vivo brain binding affinity of the PET radioligand [(18)F]-FEPPA. J. Cereb. Blood Flow. Metab. 32, 968-972 (2012).

37. Owen, D. R. et al. An 18-kDa translocator protein (TSPO) polymorphism explains differences in binding affinity of the PET radioligand PBR28. J. Cereb. Blood Flow. Metab. 32, 1-5 (2012).

38. Matthews, B. A. et al. Greater monoamine oxidase a binding in alcohol dependence. Biol. Psychiatry 75, 756-764 (2014).

39. Plane, J. M., Shen, Y., Pleasure, D. E. \& Deng, W. Prospects for minocycline neuroprotection. Arch. Neurol. 67, 1442-1448 (2010).

40. Attwells, S. et al. Inflammation in the neurocircuitry of obsessive-compulsive disorder. JAMA Psychiatry 74, 833-840 (2017).

41. Kreisl, W. C. et al. In vivo radioligand binding to translocator protein correlates with severity of Alzheimer's disease. Brain 136, 2228-2238 (2013)

42. Rusjan, P., Da Silva, T., Mizhrai, R. \& Houle, S. Accounting for endothelial binding in the kinetic modelling of [18F]FEPPA, a TSPO radioligand. Neurorecept. Mapp. 1, 350 (2018).

43. Agwuh, K. N. \& MacGowan, A. Pharmacokinetics and pharmacodynamics of the tetracyclines including glycylcyclines. J. Antimicrob. Chemother. 58 256-265 (2006).

44. Bhattacharya, A. Recent advances in CNS P2X7 physiology and pharmacology: focus on neuropsychiatric disorders. Front. Pharmacol. 9, 30 (2018).

45. Liu, C. Y., Wang, X., Liu, C. \& Zhang, H. L. Pharmacological targeting of microglial activation: new therapeutic approach. Front. Cell. Neurosci. 13, 514 (2019).

46. Dantzer, R., O'Connor, J. C., Freund, G. G., Johnson, R. W. \& Kelley, K. W. From inflammation to sickness and depression: when the immune system subjugates the brain. Nat. Rev. Neurosci. 9, 46-56 (2008). 